\title{
Ischemic Modified Albumin Predicts Critical Coronary Artery Disease in Unstable Angina Pectoris and Non-ST-Elevation Myocardial Infarction
}

\author{
Abdullah Orhan Demirtas ${ }^{\mathrm{a},}$, , Turgut Karabag ${ }^{\mathrm{b}}$, Derya Demirtas ${ }^{\mathrm{c}}$
}

\begin{abstract}
Background: In this study, whether ischemia modified albumin (IMA) has a role in showing severity or criticality of coronary arteries in patients with unstable angina pectoris (USAP)/non-ST-elevation myocardial infarction (NSTEMI) was assessed.

Methods: A total of 65 patients (40 male (M) and 25 female (F) patients; mean age $59.7 \pm 12.1$ years) with the initial diagnosis of USAP/NSTEMI were included in this study. The levels of IMA, troponin $\mathrm{T}$, creatine kinase $\mathrm{MB}$ (CK-MB), C-reactive protein (CRP), brain natriuretic peptide (BNP), creatinine, lipid panel, and whole blood count were measured from venous blood obtained from each patient within $3 \mathrm{~h}$ after the onset of symptoms. A $50 \%$ or greater coronary lumen stenosis of any coronary vessel or lateral branch was considered as critical stenosis. The severity of coronary artery disease (CAD) was assessed using the Gensini scoring system.
\end{abstract}

Results: IMA was significantly higher in patients with critical coronary artery stenosis (median, 206 vs. 23; P $<0.001$ ). There was a weak correlation between the Gensini score and IMA; whereas there was a moderate correlation between the Gensini score and BNP levels $(\mathrm{r}=0.44, \mathrm{P}=0.02)$.

Conclusion: The level of IMA can predict the criticality of CAD; however, it cannot predict the severity of CAD according to Gensini score in patients with USAP/NSTEMI.

Keywords: Acute coronary syndrome; Ischemia modified albumin; Gensini score; Coronary artery disease

Manuscript submitted March 22, 2018, accepted April 30, 2018

aDepartment of Cardiology, Health Sciences University Adana City Hospital, Adana, Turkey

bDepartment of Cardiology, Istanbul Educational Research Hospital, Istanbul, Turkey

'Department of Internal Medicine, Health Sciences University Adana City Hospital, Adana, Turkey

${ }^{\mathrm{d} C}$ Corresponding Author: Abdullah Orhan Demirtas, Department of Cardiology, Adana City Hospital, Yuregir, Adana, Turkey.

Email: aorhandemirtas@gmail.com

doi: https://doi.org/10.14740/jocmr3417w
Introduction

Acute coronary syndrome (ACS) is a manifestation of cardiac ischemia and results in myocardial injury and necrosis in line with the duration of ischemia. Cardiac markers, such as creatine kinase MB (CK-MB), troponin I and $\mathrm{T}$ are used in the diagnosis and risk stratification of patients with chest pain and suspected ACS. For these biomarkers to be positive proteins should be released from myocytes; in other words, there should be sufficient amount of cell death. Accurate and early diagnosis of ACS and early prediction of current coronary artery disease (CAD) severity, play a major role in the treatment.

Ischemia modified albumin (IMA) is a relatively new marker approved by the US Food and Drug Administration (FDA) and cleared as a risk stratification tool in myocardial ischemia and suspected ACS [1]. Sinha et al investigated whether IMA elevations correlate with increases in other markers of oxidative stress, i.e., 8-iso prostaglandin F2-A (iP) [2]. They compared IMA versus iP plasma levels in 19 patients undergoing PCI and 11 patients undergoing diagnostic angiography (controls). During PCI, all 19 patients had chest pain and 18 had transient ischemic ST segment changes. IMA was elevated from baseline in 18 of the 19 patients after PCI. Median IMA levels were higher after PCI compared with baseline. Levels remained elevated at $30 \mathrm{~min}$ and returned to baseline at $12 \mathrm{~h}$. iP levels were raised after PCI in nine of the 19 patients. However, median iP levels were not significantly different immediately or $30 \mathrm{~min}$ after PCI. In the control group, IMA and iP levels remained unchanged before and after angiography. They concluded that IMA was a more consistent marker of ischemia than iP in patients who develop chest pain and ST segment changes during PCI. Collinson et al assessed if the combination of cardiac troponin and IMA can be used for early exclusion of acute myocardial infarction (AMI) [3]. IMA or cardiac troponin was elevated in the admission sample of all patients with a final diagnosis of AMI $(n=37)$ with IMA alone elevated in $2 / 37$, cardiac troponin alone in $19 / 37$, and both in $16 / 37$. In $173 / 501$ patients in whom AMI was excluded, both tests were negative. They concluded that admission measurement of cardiac troponin plus IMA could be used for early classification of patients presenting to the emergency department to assist in patient triage. Besides its role as an efficient molecule in the diagnosis of ischemia, the detection of IMA in the early period is considered to offer more advantages compared to most mol- 
ecules [4]. Despite these advantages of IMA, its association with the severity of CAD has not yet been fully elucidated. In this study, we investigated if the levels of IMA could help predicting the severity or criticality of CAD in patients with unstable angina pectoris/non-ST-elevation myocardial infarction (USAP/NSTEMI).

\section{Materials and Methods}

\section{Patient selection}

A total of 70 patients (45 men, 25 women; mean age: $62.1 \pm$ 10.6 years) who presented to the emergency department of Bulent Ecevit University Hospital with angina or anginal equivalent complaints, ST segment depression or marked T wave inversion on ECG and/or with biomarkers of myocardial necrosis above normal levels or slightly raised and received the initial diagnosis of USAP/NSTEMI were included in this study.

The exclusion criteria included patients with myocardial infarction with ST elevation, those with symptom onset longer than $6 \mathrm{~h}$, stable angina pectoris, severe systolic heart failure (ejection fraction $<40 \%$ ), pregnant women, patients with end stage renal insufficiency, cancer, cerebrovascular stroke, pulmonary embolism and those with diseases that interfere with plasma albumin levels such as nephrotic syndrome or chronic liver disease. Standard medical treatment regimen by taking current vital parameters into account, was administered in line with current guidelines in each patient. A total of five patients who were on medications that are likely to interfere with plasma albumin levels and those in whom valvular heart disease, tachyarrhythmia, was detected, were excluded from the study. The study was completed with 65 patients ( 40 men, 25 women; mean age: $59.7 \pm 12.1$ years). All procedures were carried out in accordance with the ethical standards of the responsible committee on human experimentation (institutional and national) and with the Helsinki Declaration of 1975, as revised in 2008. All participants gave informed written consent. Local ethics committee approved the study.

\section{Coronary angiography}

Coronary angiography was performed in all patients. All angiogram runs were performed using a Philips (Integris $\mathrm{BH}$ 5000 , Philips, Netherlands) coronary angiography device. Selective coronary angiograms, performed via femoral approach using 6-French (F) and 7-F Judkins catheters, were evaluated by quantitative analysis (AET-met S.P.A. Italy QCA). A 50\% or greater coronary lumen stenosis of any coronary vessel or its lateral branch was considered as critical stenosis. The percentage of the stenosis in coronary arteries was determined by consensus of two operators. Based on these criteria, 51 of the patients had significant CAD (33 men, 18 women; mean age: $62.1 \pm 10.6$ years), and 14 had non-significant CAD (seven men, seven women; mean age: $51.3 \pm 13.8$ years).

The severity of CAD was assessed using the Gensini scoring system. Patients were classified as having mild, moder- ate and severe CAD according to the Gensini scoring system. The Gensini score was calculated by multiplying the severity coefficient, determined according to the degree of vessel narrowing by the coefficient determined based on the functional importance of the myocardial area supplied by the vessel with stenosis. According to this classification system, 1 indicates stenosis of $0-25 \%, 2$ indicates stenosis of $25-50 \%, 4$ indicates stenosis of $50-75 \%, 8$ indicates stenosis of $75-90 \%, 16$ indicates stenosis of $90-99 \%$ and 32 indicates stenosis of $100 \%$, which then was multiplied by the coefficient assigned to each main coronary artery and each segment ( 5 for the left main coronary lesion; 2.5 for the proximal left anterior descending branch and left circumflex artery; 1.5 for the mid segment of the left descending artery; 1 for the first diagonal branch and obtuse marginal branches and 1 for the right coronary artery; 0.5 for the second diagonal branch and posterolateral branch of the left circumflex artery) and the results were summed, thus giving the Gensini score for each patient. A Gensini score of 54 or greater was considered as high, a score of $24-54$ as moderate and a score below 24 as low. Patients were then separated into low, moderate and high score groups and these three groups were individually statistically analyzed except for the control group and the severe CAD group. There were 25 patients ( 13 women, 12 men; mean age: $57.7 \pm 17.7$ years) in the low Gensini score group, 24 patients (six women, 18 men; mean age: $59.4 \pm 9.5$ years) in the moderate score group and 16 patients (six women, 10 men; mean age: $62.8 \pm 9.1$ years) in the high score group.

\section{Laboratory parameters}

The levels of troponin T, CK-MB, C-reactive protein (CRP), brain natriuretic peptide (BNP), creatinine, lipid panel, and whole blood count were measured from venous blood obtained from each patient within $3 \mathrm{~h}$ after the onset of symptoms. Venous blood samples obtained for the measurement of levels of IMA were centrifuged at 4,000 rpm for $10 \mathrm{~min}$ and stored at $-80{ }^{\circ} \mathrm{C}$ until needed. Serum IMA was analyzed using an IMA ELISA kit (E90825Hu 96 Tests, Enzyme-linked Immunosorbent Assay Kit for Ischemia Modified Albumin, China).

\section{Statistical analysis}

Statistical analysis was performed using SPSS for Windows 13.0. The Kolmogorov-Smirnov test was applied to test for a normal distribution of numerical variables. Descriptive statistics for numerical variables are presented as mean (maximalminimal value); whereas categorical data are presented as numbers and percentage. The Chi-square test and Fisher exact Chi-square test were used to analyze the differences between the groups regarding categorical variables. Regarding numerical variables, comparison of means between the control and critical CAD groups and the low, moderate, high score groups was performed using the significance test when the assumptions of parametric test assumptions were met and applying Mann-Whitney U test when the assumptions of parametric test assumptions were not met. A regression analysis was used to analyze the relationship between IMA levels and the severity 
Table 1. Comparison of Demographic Characteristics, IMA, CRP, BNP and Troponin Levels Between Patients With Critical CAD and Non-Critical CAD

\begin{tabular}{llll} 
& Critical CAD $(\mathbf{n}=\mathbf{5 1})$ & Non-critical CAD (n= 14) & P $(\mathbf{P}<\mathbf{0 . 0 5})$ \\
\hline Age (years) & $64(41-82)$ & $45.5(30-77)$ & $0.020^{*}$ \\
Gender (n) & $18 \mathrm{~W}, 33 \mathrm{M}$ & $7 \mathrm{~W}, 7 \mathrm{M}$ & 0.489 \\
Diabetes mellitus (n) & 21 & 4 & 0.583 \\
Hypertension (n) & 32 & 8 & 0.943 \\
Hyperlipidemia (n) & 17 & 3 & 0.521 \\
Smoking (n) & 20 & 5 & 0.625 \\
Systolic blood pressure (mm Hg) & $130(100-180)$ & $125(100-142)$ & 0.194 \\
Diastolic blood pressure (mm Hg) & $80(50-100)$ & $70(50-90)$ & $0.006^{*}$ \\
Familial history of CAD (n) & 12 & 5 & 0.493 \\
Heart rate (beats/min) & $83(59-120)$ & $76.5(57-108)$ & 0.155 \\
IMA (ng/mL) (median) & $206(8-1,131)$ & $23(2-123)$ & $<0.001 *$ \\
CRP (mg/dL) & $3.8(1-97)$ & $1.1(1-18.5)$ & 0.076 \\
BNP (pg/mL) & $90(3-614.6)$ & $36.4(7.1-121.3)$ & $0.012 *$ \\
Troponin (ng/mL) & $0.03(0-4.1)$ & $0.01(0.1-2.3)$ & 0.632 \\
\hline
\end{tabular}

BNP: brain natriuretic peptide; CAD: coronary artery disease; CRP: C-reactive protein; IMA: ischemia modified albumin; M: men; W: women. *Age and diastolic blood pressure were significantly higher in the critical CAD group compared to the noncritical CAD group. The comparison of patients with and without critical CAD revealed that IMA and BNP levels were significantly higher in patients with critical CAD.

of CAD in all patients and each group individually. The relationship between IMA levels and Gensini scores was analyzed using a correlation analysis. A P value of $<0.05$ was considered statistically significant with a confidence interval of $95 \%$. Receiver operating characteristic (ROC) curve was plotted to determine the best diagnostic cut-off value for IMA.

\section{Results}

Patients were divided into two groups: critical CAD group and non-critical CAD group (control). There were 51 patients with critical CAD (33 men, 18 women; mean age: $62.1 \pm 10.6$ years) and 14 with non-critical CAD (seven men, seven women; mean age: $51.3 \pm 13.8$ years). The critical CAD group and the control group were similar in terms of systolic blood pressure, heart rate, gender, diabetes mellitus, hypertension, hyperlipidemia, familial history of CAD, and smoking history. Age and diastolic blood pressure were significantly higher in the critical CAD group compared to the noncritical CAD group $((64$ (41 - 82) vs. $45.5(30-77), \mathrm{P}=0.02 ; 80(50-100)$ vs. 70 (50 - 90), $\mathrm{P}=0.006)$ (Table 1)).

Patients with a score below 24 were classified as having low, those with a score between 24 and 54 as having moderate and those with a score of 54 or greater as having high Gensini scores. The classification of the groups according to Gensini score level revealed that 25 patients ( 13 women, 12 men; mean age: $57.7 \pm 17.7$ years) had low, 24 patients (six women, 18 men; mean age: $59.4 \pm 9.5$ years) had moderate and 16 patients (six women, 10 men; mean age: $62.8 \pm 9.1$ years) had high Gensini scores. There were no differences in demographic characteristics between these three groups (Table 2).
The classification of the groups according to both the presence of critical CAD and Gensini score showed no significant differences in levels of creatinine, uric acid, fasting blood glucose, low-density lipoprotein (LDL), high-density lipoprotein (HDL), triglyceride, total cholesterol, CK-MB, and whole blood count (white blood cell (WBC), hemoglobin, hematocrit, platelet count, and mean platelet volume).

Nineteen patients had a troponin value above $0.06 \mathrm{ng} / \mathrm{mL}$, defined as the upper reference limit in our center; whereas 46 patients had a troponin value below $0.06 \mathrm{ng} / \mathrm{mL}$. BNP was above the upper reference limit in 50 patients; whereas 15 patients had a BNP level below the upper reference limit.

The comparison of patients with and without critical CAD revealed that IMA and BNP levels were significantly higher in patients with critical CAD (Table 1). Levels of CRP and troponin were also higher in those with critical CAD, which however did not reach the level of statistical significance. The classification of the groups according to the Gensini score level showed that BNP and troponin levels were significantly higher in the high Gensini score group compared to the other groups (Table 2). The levels of IMA and CRP were similar between the groups.

The correlation analysis revealed a weak correlation between the Gensini score and IMA and age $(\mathrm{r}=0.25, \mathrm{P}=0.05$; $\mathrm{r}=0.28, \mathrm{P}=0.03$, respectively) and a moderate correlation between the Gensini score and BNP levels $(r=0.44, \mathrm{P}=0.02)$.

The cut-off value for IMA in diagnosing critical CAD was 123. Values of 123 or greater predicted critical coronary artery stenosis with a sensitivity of $64.7 \%$ and a specificity of $78.6 \%$ (area under curve: 0.704; Fig. 1).

IMA levels were investigated in patients with troponin levels above and below the upper reference limit, but no dif- 
Table 2. Demographic and Laboratory Characteristics of the Patients According to Gensini Scores

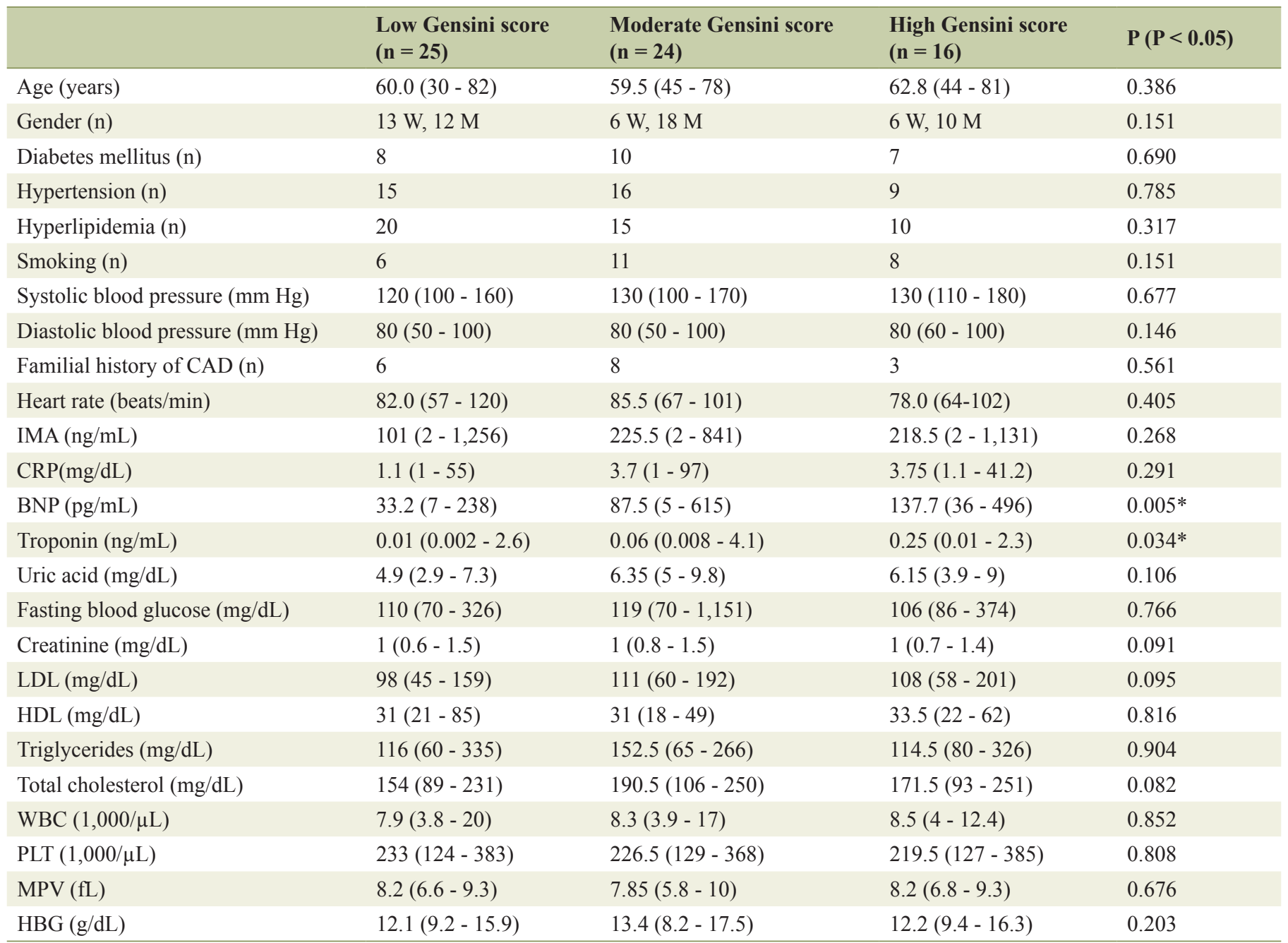

BNP: brain natriuretic peptide; CAD: coronary artery disease; CRP: C-reactive protein; IMA: ischemia modified albumin; LDL: low-density lipoprotein; HDL: high-density lipoprotein; WBC: white blood cell; PLT: platelet; MPV: mean platelet volume; HBG: hemoglobin. *The groups according to the Gensini score level showed that BNP and troponin levels were significantly higher in the high Gensini score group compared to the other groups.

ference was noted.

\section{Discussion}

The prediction of the CAD severity or criticality in ACS can be useful in the choice of treatment approach. Therefore, recent researches have focused on identifying a non-invasive and easily available cardiac marker that can yield results rapidly particularly in emergency setting. An optimum marker should enable the prediction of ischemia independent from myocardial injury, be elevated in the early period, circulate in blood long enough to enable its detection, be easily measured, be costeffective, have high specificity and sensitivity and come back in normal range in a $24-\mathrm{h}$ period, thus enabling the prediction of a recurrent ischemic attack [5].

Previous studies have shown that serum albumin undergoes conformational transitions under ischemic conditions. N- terminal region of normal human albumin detoxifies free oxygen radicals and is a binding site for metals specifically cobalt, copper, and nickel. During ischemia, oxygen radicals damage the N-terminal region of human albumin and decrease albumin's capacity to bind metals. This modified albumin is referred to as IMA. Elevation of IMA is directly associated with the amount of free oxygen radicals that form during ischemia [6].

IMA has also been reported to increase not only in coronary ischemia but also in ischemic conditions such as cerebrovascular diseases and pulmonary embolism [7-11]. In a study, Lippi et al suggested that in vivo generation of IMA was an endogenous mechanism of response to ischemia, preventing myocardial injury and limiting the extent of myocyte necrosis [12]. Clinical studies have shown that IMA levels are increased shortly after the onset of ischemia, maintain these levels for $6-12 \mathrm{~h}$ and then return to basal levels in $24 \mathrm{~h}$ [5], which indicates that IMA is a marker that can be used both in the early and late periods. Even though IMA was significantly higher in patients with criti- 


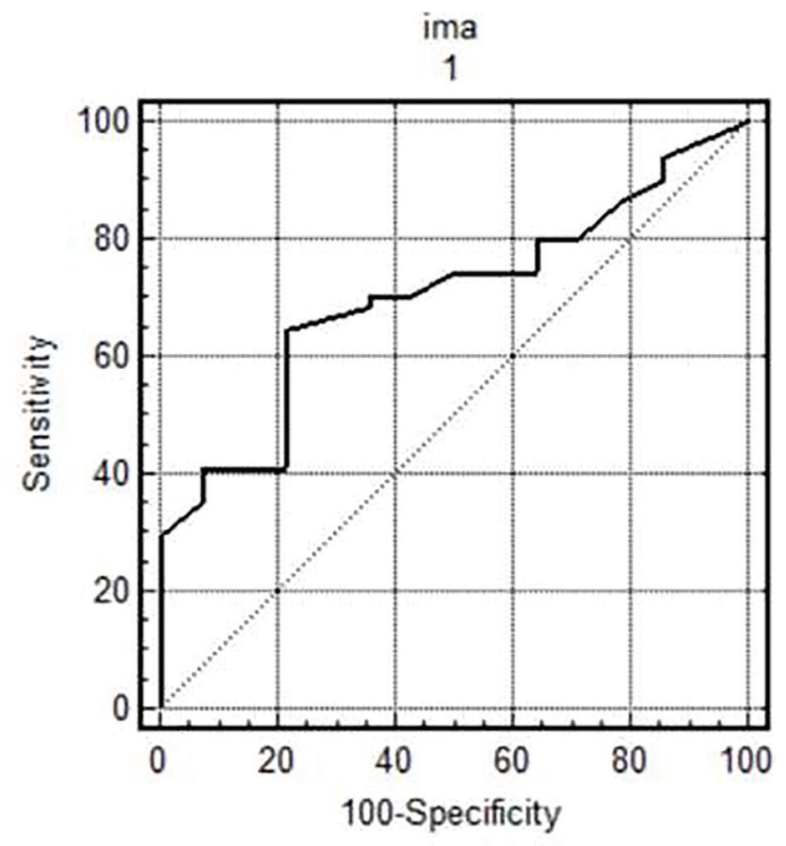

Figure 1. IMA cut-off value in diagnosing critical coronary artery disease. Values of 123 or greater predicted critical coronary artery stenosis with a sensitivity of $64.7 \%$ and a specificity of $78.6 \%$ (area under curve: 0.704).

cal CAD in this study, IMA levels were similar among patients with different Gensini scores. As is well known, the presence of extensive disease in a coronary artery may not always result in ischemia. The development of ischemia is associated with severely narrowed coronary arteries with active plaque and inflammation and thrombus burden of the active plaque. The regression analysis demonstrated that IMA was associated not with the Gensini score but with whether the disease was critical or not, which indicated that the presence of critical CAD could be predicted in patients with elevated IMA levels. In addition, the detection of high IMA levels in patients presenting with clinical signs and symptoms of ACS with no ST elevation to the emergency department was indicative of critical CAD, requiring the consideration of invasive procedure in these patients.

Wudkowska et al investigated the usefulness of IMA measurement to differentiate patients with ACS with no ST elevation and assessed IMA levels in two study groups: one consisting of patients with elevated troponin concentration and one consisting of those with troponin concentration below reference value. They reported that the IMA concentration did not differ between patients with NSTEMI and those with USAP. On the other hand, the authors also suggested that the IMA concentration was closely associated with the duration of chest pain and correlated with BNP levels [13]. Patients with NSTEMI/USAP who presented to the emergency department within $3 \mathrm{~h}$ after the onset of chest pain were included in this study presented here. In our study, IMA levels were investigated in patients with troponin levels above and below the upper reference limit, but no difference was noted consistent with the findings of Wudowska et al. IMA levels were significantly higher in patients with critical CAD.
The cut-off value for IMA in diagnosing critical CAD was 123. Values of 123 or greater predicted critical coronary artery stenosis with a sensitivity of $64.7 \%$ and a specificity of $78.6 \%$ (area under curve: 0.704 ). ROC curve indicated best sensitivity and 1-specificity values at a cut-off of 123 . Despite its relatively low sensitivity, this cut-off value has a specificity of $78.6 \%$. With its nearly $80 \%$ specificity, it provides an advantage of diagnosis of critical coronary ischemia without any additional laboratory tests in most patients.

\section{Study limitations}

The main limitation of our study was the limited number of cases that reduces the sensitivity and specificity of multivariate analysis. One of the limitations was that the study group was confined to patients with NSTEMI/USAP. Therefore, our findings cannot be generalized to include all patients with ACS. The other limitations include our inability to demonstrate current ischemic load by using methods such as fractional flow rate, so we were unable to quantify ischemia. Due to the availability of limited number of kits in our facility, we were unable to identify a cut-off value for IMA in a large normal patient population under our own conditions.

\section{Conclusion}

IMA and BNP levels are higher in patients with critical CAD presenting with clinical signs and symptoms of USAP/NSTEMI. On the other hand, IMA levels cannot be used to predict the Gensini score of CAD. The elevated BNP level is associated with critical CAD and can predict high Gensini score without hemodynamic changes. Future large-scale comparative studies are warranted for investigating the relationship between IMA and CAD severity and criticality.

\section{Acknowledgments}

The statistical analysis of this study was carried out by Cagatay Buyukuysal in the Statistics Department of Bulent Ecevit University.

\section{Conflict of Interest}

None.

\section{Funding}

None.

\section{References}

1. Peacock F, Morris DL, Anwaruddin S, Christenson RH, 
Collinson PO, Goodacre SW, Januzzi JL, et al. Metaanalysis of ischemia-modified albumin to rule out acute coronary syndromes in the emergency department. Am Heart J. 2006;152(2):253-262.

2. Sinha MK, Gaze DC, Tippins JR, Collinson PO, Kaski JC. Ischemia modified albumin is a sensitive marker of myocardial ischemia after percutaneous coronary intervention. Circulation. 2003;107(19):2403-2405.

3. Collinson PO, Gaze DC, Bainbridge K, Morris F, Morris B, Price A, Goodacre S. Utility of admission cardiac troponin and "Ischemia Modified Albumin" measurements for rapid evaluation and rule out of suspected acute myocardial infarction in the emergency department. Emerg Med J. 2006;23(4):256-261.

4. Chawla R, Goyal N, Calton R, Goyal S. Ischemia modified albumin: A novel marker for acute coronary syndrome. Indian J Clin Biochem. 2006;21(1):77-82.

5. Lippi G, Montagnana M, Salvagno GL, Guidi GC. Potential value for new diagnostic markers in the early recognition of acute coronary syndromes. CJEM. 2006;8(1):2731.

6. Roy D, Quiles J, Gaze DC, Collinson P, Kaski JC, Baxter GF. Role of reactive oxygen species on the formation of the novel diagnostic marker ischaemia modified albumin. Heart. 2006;92(1):113-114.

7. Gunduz A, Turedi S, Mentese A, Altunayoglu V, Turan I,
Karahan SC, Topbas M, et al. Ischemia-modified albumin levels in cerebrovascular accidents. Am J Emerg Med. 2008;26(8):874-878.

8. Abboud H, Labreuche J, Meseguer E, Lavallee PC, Simon O, Olivot JM, Mazighi M, et al. Ischemia-modified albumin in acute stroke. Cerebrovasc Dis. 2007;23(23):216-220.

9. Talwalkar SS, Bon Homme M, Miller JJ, Elin RJ. Ischemia modified albumin, a marker of acute ischemic events: a pilot study. Ann Clin Lab Sci. 2008;38(2):132-137.

10. Kaya Z, Kayrak M, Gul EE, Altunbas G, Toker A, Kiyici A, Gunduz M, et al. The role of ischemia modified albumin in acute pulmonary embolism. Heart Views. 2014;15(4):106-110.

11. Turedi S, Gunduz A, Mentese A, Topbas M, Karahan SC, Yeniocak S, Turan I, et al. The value of ischemia-modified albumin compared with d-dimer in the diagnosis of pulmonary embolism. Respir Res. 2008;9:49.

12. Lippi G, Montagnana M, Guidi GC. Albumin cobalt binding and ischemia modified albumin generation: an endogenous response to ischemia? Int J Cardiol. 2006;108(3):410411.

13. Wudkowska A, Goch J, Goch A. Ischemia-modified albumin in differential diagnosis of acute coronary syndrome without ST elevation and unstable angina pectoris. Kardiol Pol. 2010;68(4):431-437. 